\title{
Electromagnetic Coupler in Wireless Charging for Electric Vehicles
}

\author{
Yufeng Liu ${ }^{1, a}$ and Chunyan Xiao ${ }^{2, b}$ \\ ${ }^{1}$ School of Automation Science and Electrical Engineering, Beihang University, Beijing 100191, \\ China \\ aliuyufeng@buaa.edu.cn, bxiao_chunyan@buaa.edu.cn
}

Keywords: Electric vehicle, Wireless charging; Electromagnetic coupler; Misalignment tolerance; Power stabilization

\begin{abstract}
The transmission power in wireless charging for electric vehicles is unstable due to the decline of the coupling coefficient caused by the misalignment of the electromagnetic coupler. Considering misalignment tolerance and coupling coefficient, the paper designed a kind of asymmetric electromagnetic coupler with large lateral misalignment tolerance. The coupling coefficient of charging pads can be greatly improved by using the asymmetric structure, and the requirement of electromagnetic safety can still be met. The rectangular coil and the linearly-arranged ferrite strip along the long side ensure that the coupler has greater lateral misalignment tolerance, which solves the problem of not easy adjustment of lateral misalignment when electric vehicles park for wireless charging. The experimental results of transmission power with misalignment well agree with simulation results. Both simulation and experimental results show that the transmission power can keep stable within a wide offset range. In experiments, the transmission power of $2.1 \mathrm{~kW}$ at the interval of $125 \mathrm{~mm}$ has been realized, and the power variation within the offset of $100 \mathrm{~mm}$ is no more than $20 \%$.
\end{abstract}

\section{Introduction}

Electric Vehicles (EV) has been rapidly developed due to its green environmental protection, zero emission. At present, Electric Vehicles are mainly charged with cables, but it will bring with some troubles, such as sparks, wear and tear, unsafe and hard to maintain. Wireless Power Transfer(WPT) can get rid of the dependence on cables, which can overcome all disadvantages above, and make the power supply for EV has a new choice[1].

Wireless Power Transfer system is mainly composed of four parts: power supply with high frequency, compensation network, electromagnetic coupler and load. In this paper, the single transmitting or receiving device is named as power pad, electromagnetic coupler is composed of transmitting and receiving power pad. The transmission power and efficiency are mainly depend on power supply, compensation network, circuit parameters, magnetic field of electromagnetic coupler and load[2-6]. In WPT system, the coupling coefficient between two power pads influence the circuit parameter design directly, it is one of the decisive factors of transmission efficiency, transmission distance and misalignment tolerance [7-9]. Electromagnetic coupler, as the key component of the wireless power transfer system, has been paid more attention by researchers, and has been continuously studied and applied.

For the electric vehicle wireless charging system, due to the working state of the charging battery and charging time and other restrictions, the required transmission power should be kept in a certain range, that is, to maintain the stability of the transmission power. There are many solutions to this problem[10-13]. In mobile system, quadrature winding was proposed in [10], it can capture both vertical and lateral magnetic flux to increase misalignment tolerance. The misalignment tolerance was defined in order to study the influence of misalignment of different electromagnetic coupler (coupling coefficient variation) on the transmission power. Misalignment tolerance is defined as the misalignment when the coupling coefficient of the electromagnetic coupler is reduced to $80 \%$ of the maximum coupling coefficient. Polyphone systems have been developed in [11]. In battery charge 
systems for idle vehicles, Position detection and mechanical positioning system was used in [12] for vehicle positioning, making the two coils on the right position, so as to ensure the transmission power. the SPS topology is proposed in [13], which is suitable for mobile system battery charge, where high misalignment may occur. In summary, in order to ensure the stability of transmission power, the existing literature gives solutions mainly from two aspects. One is to maintain the stability of the coupling coefficient, that is, to maintain the coupling coefficient in a certain range; two is to adopt new type of compensation network. Although the new compensation network can maintain the stability of the power when the coupling coefficient is reduced, it will also reduce the transmission efficiency. Position detection and mechanical positioning system was effective, but it will increase the complexity of the whole system.

In this paper, the coupler was optimized, and the coupling coefficient can be stable when high misalignment happened, so the transmission power can be kept stable. Firstly, the relationship between the transmission power and factors of the WPT system is studied. Secondly, the basic structure was designed based on the actual needs of EV charging, the coupling coefficient of the circular and other three couplers with this structure are analyzed by simulation, the lateral rectangular power pad with the largest misalignment was chosen. Then, according to the actual demand of the electric vehicle, the parameters of the lateral rectangular power pad are adjusted, and the simulation results show that it has a large misalignment tolerance. Finally, on the experimental setup, the transmission power is measured under different misalignment, which verifies that the designed electromagnetic coupler has a large misalignment tolerance.

\section{Transmission Power and Its Influencing Factors of WPT}

In WPT system for EV, there are many factors that affect the transmission power, including the power parameters, the circuit component parameters, the two charging plate coupling coefficient, the compensation network, load characteristics, etc [4, 5]. In the following, compensation network will be analyzed at first, then, the relationship between the transmission power and the coupling coefficient of the electromagnetic coupler is studied.

In WPT system, when the whole system is under resonant state, the transmission efficiency and power will be greatly improved. The commonly used compensation networks are series-series (SS) [3, $9,14]$ and double-sided LCC compensation $[4,15,16]$. The structure of SS compensation is simple, but if two power pads are not aligned correctly, the coupling coefficient will be very small, the output current is too large to destroy the power supply. Double-sided LCC compensation network has two advantages: First, under resonant state, when the coupling coefficient is small, the output current will also be small, the power supply can be protected. Second, the current on primary coil is independent of coupling coefficient and load, which makes the induced voltage on secondary side is stable. But the double-sided LCC compensation is much more complex than SS compensation, it needs more elements, and the actual resonant impedance matching is more difficult. Combining the advantages of two kinds of compensation network, LCC-C compensation network is shown in Fig. 1, LCC compensation is used in primary side to protect the power supply and make the current on transmitting stay constant, and series compensation is used on secondary side to make resonant tuning convenient.

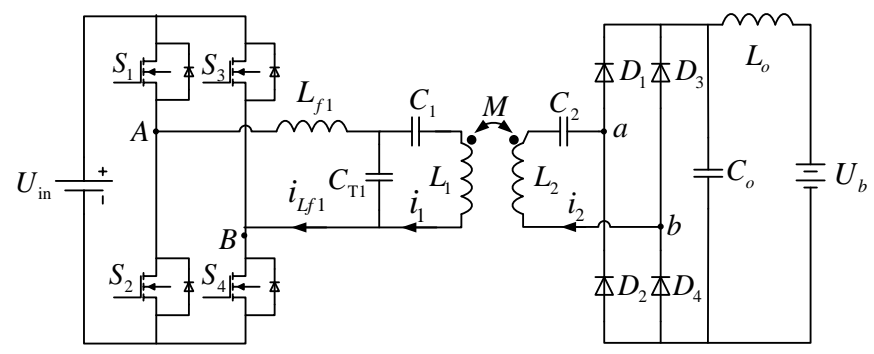

Figure 1. Wireless charging system circuit model with LCC-C compensation circuit 
The circuit model of WPT with LCC-C compensation circuit is shown in Fig. 1. $S_{1} \sim S_{4}$ are four MOSFETs in the primary side, $D_{1} \sim D_{4}$ are rectifier diodes in the secondary side, $L_{1}$ and $L_{2}$ are the self-inductance of the transmitting and receiving coil, $L_{f 1}, C_{\mathrm{T} 1}$ and $C_{1}$ are the primary-side compensation inductor and capacitor, $C_{2}$ is the secondary-side compensation capacitor. $M$ is the mutual inductance between two coils. $u_{A B}$ is the input voltage applied on the compensated circuit, $u_{a b}$ is the output voltage before the rectifier diodes. $i_{1}, i_{2}, i_{L f 1}$ are the currents on $L_{1}, L_{2}, L_{f 1}$. $\dot{\mathrm{U}}_{A B}, \dot{\mathrm{U}}_{a b}$, $\dot{\mathrm{I}}_{1}, \dot{\mathrm{I}}_{2}$ and $\dot{\mathrm{I}}_{L f 1}$ represent for the phasor form of the corresponding variables. $R_{\text {eq }}$ represent for the equivalent resistance consist of rectifier, filter and battery.

Ignore the contact resistance of the transmitting and the receiving coil, the equivalent circuit model can be established as shown in Fig. 1. When the system is in the resonance state, the current and transmission power $\mathrm{P}$ can be expressed as following:

$$
\begin{aligned}
& \dot{\mathrm{I}}_{L f 1}=\left(\frac{M}{L_{f 1}}\right)^{2} \frac{\dot{\mathrm{U}}_{A B}}{R_{\mathrm{eq}}} \\
& \dot{\mathrm{I}}_{1}=\frac{\dot{\mathrm{U}}_{A B}}{\mathrm{j} \omega_{0} L_{f 1}} \\
& \dot{\mathrm{I}}_{2}=\frac{M}{L_{f 1} R_{\mathrm{eq}}} \dot{\mathrm{U}}_{A B} \\
& P=\dot{\mathrm{U}}_{A B} \dot{\mathrm{I}}_{L f 1}=k^{2} \cdot \frac{L_{1} L_{2}}{L_{f 1}{ }^{2}} \cdot \frac{U_{A B}^{2}}{R_{\mathrm{eq}}}
\end{aligned}
$$

It can be seen from (4) that transmission power is related to many factors, including the input voltage $U_{A B}$, load $R_{\text {eq }}$, inductance $L_{f 1}, L_{1}$ and $L_{2}$, as well as the coupling coefficient $k$. The transmission power is proportional to the square of the coupling coefficient, the misalignment of the vehicle leads to the decrease of the coupling coefficient, which will make the transmission power decrease rapidly. Therefore, to maintain the stability of the transmission power in a large misalignment range, it is necessary to stabilize the coupling coefficient $k$, that means a large misalignment tolerance.

\section{Structure of Electromagnetic Coupler}

The following will be based on the requirements of EV in wireless charging, a coupler which can stabilize the coupling coefficient in a large misalignment range will be designed.

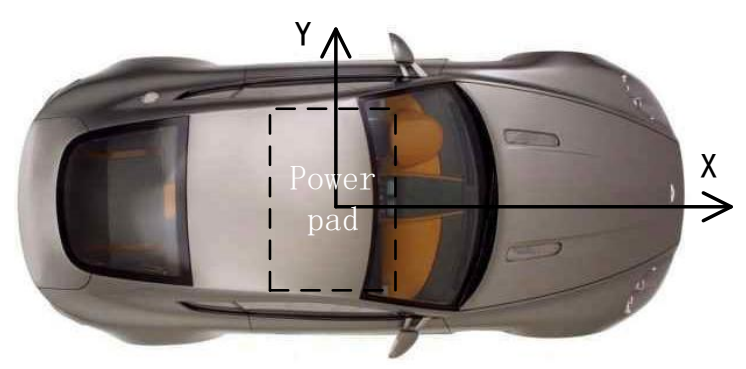

Figure 2. Two offset directions of EV

There are two types of misalignment as illustrated in Fig. 2: X direction, also named front-to-rear direction, $\mathrm{Y}$ direction, also named door-to-door direction. When $\mathrm{X}$ direction misalignment happened, it is much easier for drivers to adjust with the help of simple detection signals. But when Y direction misalignment happened, it is not convenient to adjust. Therefore, the $\mathrm{Y}$ direction misalignment should be paid more attention in the design of power pad. Circular coupler has the same misalignment tolerance in all directions, increasing the size can increase the misalignment tolerance in each direction, this will cause a waste in $\mathrm{X}$ direction misalignment tolerance and increase cost. Therefore we propose a rectangular power pad which has different misalignment tolerance in different direction. 
The better misalignment tolerance will be chosen for Y direction misalignment, and the worse one can be chosen for the $\mathrm{X}$ direction misalignment.

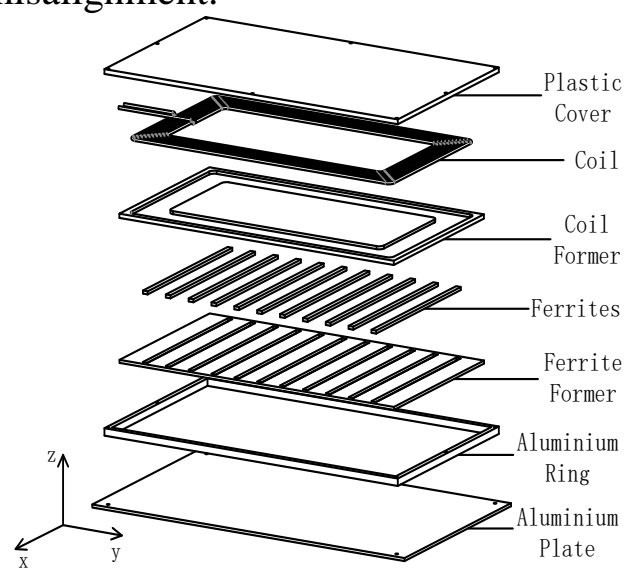

Figure 3. Power pad with rectangular structure

The structure of rectangular power pad is shown as Fig. 3. The power pad is mainly composed of plastic cover, coil, coil former, ferrites, ferrite former, aluminium ring, aluminium plate. Aluminium plate is used for fixing and shielding magnetic flux leakage. The ferrite strips are used for guiding magnetic field produced by the coil. Coil former and ferrite former are used for fixing coil and ferrite, the plastic cover is used for packaging the whole power pad. Ferrite strips are used instead of ferrite plate, that is because it can weaken the magnetostrictive effect, and the production process of strip is simple.

\section{Relationship between Misalignment and Coupling Coefficient of Four Kinds of Power Pad}

Circular coupler is a kind of typical unipolar coupler[17], in the followings, circular pad will be regarded as reference standard, according to the principle that the area of power pad and the volume of magnetic material is equivalent, three kinds of power pad are designed according to the power pad structure in Fig. 3, and compared with circular one in simulation.

Table 1 Parameters of four kinds of power pad

\begin{tabular}{ccccc}
\hline & $\begin{array}{c}\text { Simulation } \\
\text { model }\end{array}$ & $\begin{array}{c}\text { Number } \\
\text { of } \\
\text { ferrite }\end{array}$ & $\begin{array}{c}\text { Ferrite } \\
\text { size/mm }\end{array}$ & $\begin{array}{c}\text { Coil } \\
\text { size/mm }\end{array}$ \\
\hline $\begin{array}{c}\text { Circular } \\
\text { pad }\end{array}$ & 8 & $150 \times 15 \times 5$ & $\phi 500$ \\
$\begin{array}{c}\text { Square pad } \\
\text { Vertical } \\
\text { rectangular } \\
\text { pad } \\
\text { Lateral } \\
\text { rectangular } \\
\text { pad }\end{array}$ & 6 & $600 \times 5 \times 5$ & $600 \times 327 \times 4$ \\
\hline & 11 & $327 \times 5 \times 5$ & $327 \times 600 \times 4$ \\
\hline
\end{tabular}

Four types of power pad are shown in Table 1. Vertical rectangular pad and Lateral rectangular pad represent for the longer vertical side pad and longer lateral side pad, the air gap between two power pads are $175 \mathrm{~mm}$. Finite Element Analysis will be used to simulate the relationship between coupling coefficient and misalignment. 


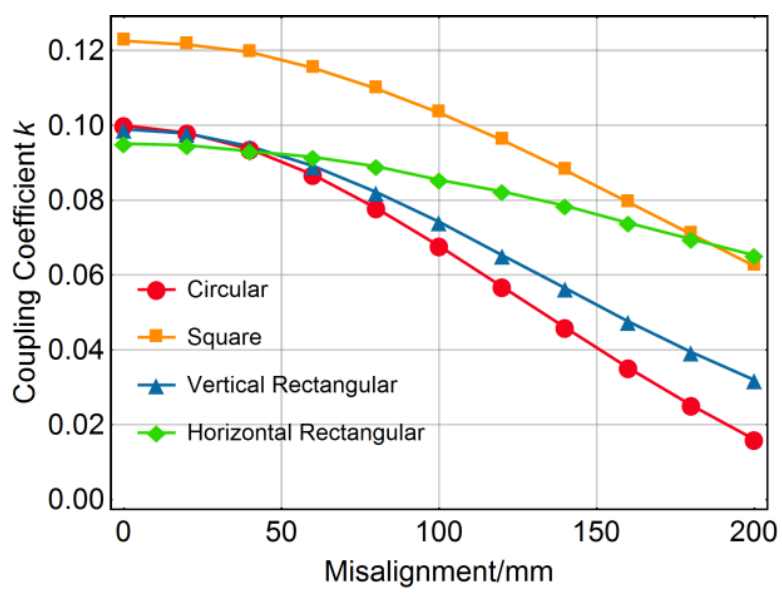

Figure 4. Coupling coefficients versus misalignments of four kinds of power pads

The relationship between the coupling coefficient and misalignment is shown in Fig. 4. As can be seen from the figure, when the misalignment is small, the coupling coefficient of square power pad is the largest, the other three kinds of power pad is near to each other. With the increase of misalignment, the coupling coefficient of the circular pad dropped greatly, and then decent speed of square pad is close to the vertical rectangle pad, the coupling coefficient of lateral rectangular power pad dropped slowly, the misalignment tolerance of each pad is $60 \mathrm{~mm}, 100 \mathrm{~mm}, 80 \mathrm{~mm}, 140 \mathrm{~mm}$. Although the coupling coefficient of square power pad is larger than lateral rectangular power pad in a large misalignment range, the coupling coefficient of the lateral rectangular power pad is more stable with the change of the misalignment. For example, if the transmission power of WPT system is $2 \mathrm{~kW}$, according to the relationship between coupling coefficient and transmission power as shown in (4), when the misalignment is $200 \mathrm{~mm}$, transmission power of square power pad will decrease to $500 \mathrm{~W}$, however, the transmission power of lateral rectangular power pad will only decrease to nearly $1000 \mathrm{~W}$, that is to say the transmission power of lateral rectangular power pad is much stable than other three when misalignment happened.

\section{Experiment and Result Analysis}

The structure of power pad above designed is symmetrical, the structure of transmitting and receiving power pad are all the same, all of which are shielded by metal plate. The receiving power pad adopts the aluminum plate to shield the magnetic flux leakage, which can reduce the eddy current in the bottom plate of the EV, and the system can satisfy the requirement of electromagnetic safety of human body. Therefore, the shield metal plate of the power pad is changed into a non metal plate in the design, and the shielding material of the coupler is asymmetrical, so it was named asymmetrical coupler.

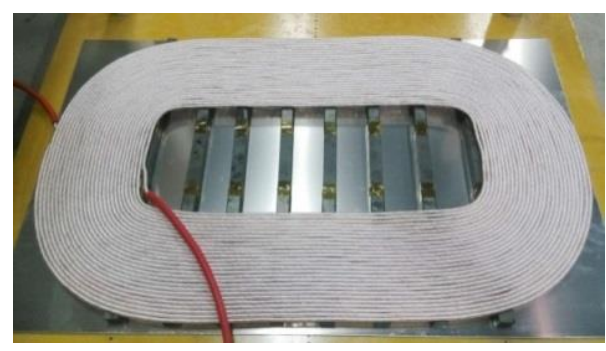

Figure 5. Power pad for electric vehicle

Considering the requirement of the installation space of $\mathrm{EV}$ and the transmission power, the power pad is designed as shown in Fig. 5. The size of coil is $380 \mathrm{~mm} \times 620 \mathrm{~mm}$, it was made by Litz 
wire(AWG38-800 strands). The number of ferrite bar is 8 , the size of it is $375 \mathrm{~mm} \times 15 \mathrm{~mm} \times 15 \mathrm{~mm}$, it is built from small ferrite pieces(TDK PC40 $85 \mathrm{~mm} \times 15 \mathrm{~mm} \times 5 \mathrm{~mm}$ and TDK PC40 $60 \mathrm{~mm} \times 15$ $\mathrm{mm} \times 5 \mathrm{~mm}$ ). The air gap between two power pads is $125 \mathrm{~mm}$.

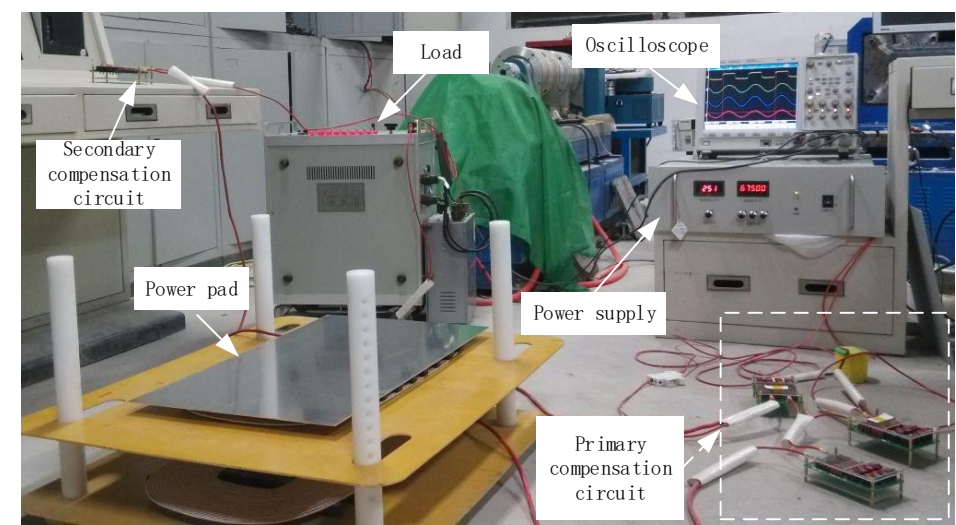

Figure 6. Experimental setup of wireless power transfer

The circuit was connected according to Fig. 1, the rectifier will be replaced by load. The experimental setup was shown in Fig. 6, and System parameters are shown in Table. 2

Table.2 System parameters

\begin{tabular}{ccc}
\hline Parameter & Value \\
\hline Input DC voltage $\mathrm{U}_{\text {in }}$ & $210 \mathrm{~V}$ \\
Load & $37 \Omega$ \\
Nominal gap & $125 \mathrm{~mm}$ \\
Coupling coefficient & $0.29 \sim 0.33$ \\
Transmitting coil inductance & \multicolumn{2}{c}{$518.8 \mu \mathrm{H}$} \\
Receiving coil inductance & $448 \mu \mathrm{H}$ \\
Switching frequency & \multicolumn{3}{c}{$67.3 \mathrm{kHz}$} \\
Transmission power & Theoretical value $2 \mathrm{~kW}$ \\
\hline \multicolumn{3}{c}{ Actual value } \\
$L_{f 1}$ & $118.8 \mu \mathrm{H}$ & $118.8 \mu \mathrm{H}$ \\
$C_{\mathrm{T} 1}$ & $47.1 \mathrm{nF}$ & $47.3 \mathrm{nF}$ \\
$C_{1}$ & $13.98 \mathrm{nF}$ & $14.18 \mathrm{nF}$ \\
$C_{2}$ & $12.5 \mathrm{nF}$ & $12.47 \mathrm{nF}$ \\
\hline
\end{tabular}




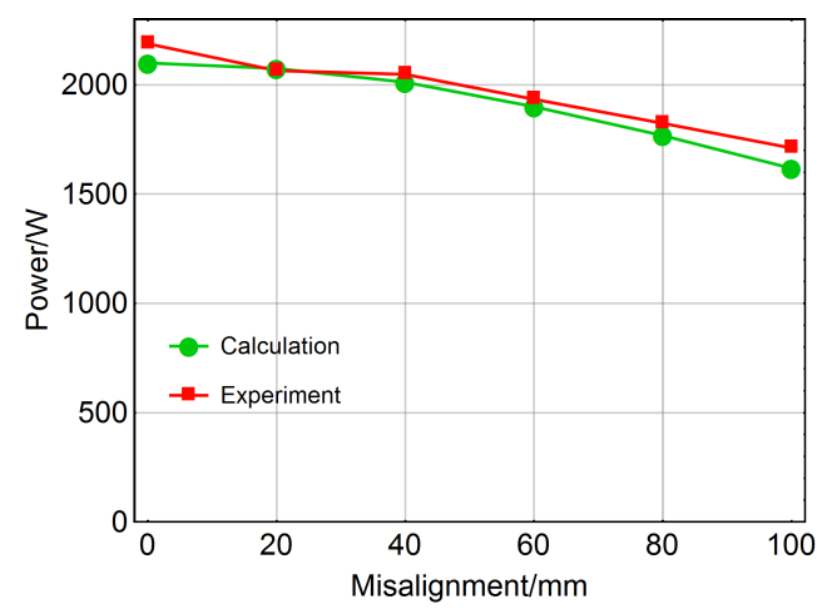

Figure 7. Transmission power versus misalignment

The relationship between transmission power and misalignment is shown in Fig. 7. In the experiment, the measured power is in good agreement with the calculated values. When misalignment increases, transmission power varies very little, due to the change of its coupling coefficient is very small. When two coils are perfectly aligned, the transmission power is $2.1 \mathrm{~kW}$, and it decreased to $1.7 \mathrm{~kW}$ at $100 \mathrm{~mm}$ misalignment, it is less than $20 \%$ compared to perfectly aligned position.

\section{Conclusion}

The transmission power shoule be stable to satisfy the requirements for wireless charging for EVs. Considering the lateral misalignment is hard to adjust, In this paper, the coupling coefficient and misalignment tolerance are design objectives, and a lateral rectangular asymmetric electromagnetic coupler with large lateral misalignment tolerance is developed. The coupler has the following characteristics: (1) the structure of coupler is asymetric, the shield metal plate of transmitting power pad is changed into a non metal plate, which can greatly increase the coupling coefficient between two pads, and still satisfy the requirement of electromagnetic safety of human body; (2) The shape of coil is rectangular, ferrite strips are placed along longer side. Simulation and experiment show that the coupler has a larger misalignment tolerance in the direction perpendicular to ferrite strips, which can make transmission power quite stable in a large misalignment range. About $2.1 \mathrm{~kW}$ was transfered through the air gap of $125 \mathrm{~mm}$ when two coils are perfectly aligned, transmission power within the $100 \mathrm{~mm}$ misalignment is not more than $20 \%$.

\section{Acknowledgements}

Project Supported by Science and Technology Foundation of State Grid Corporation of China(SGTYHT/14-JS-188)

\section{References}

[1] Yang Qingxin, Zhang Xian, Li Yang, et al. Wireless Power Transmission Technology And Its Applications (1st edition) [M]. Beijing: China Machine Press.

[2] Zhang Xian, Yang Qingxin, Cui Yulong. Design Optimization and Verification on the Power Transmitting Coil in the High-Power Wireless Power Transmission System[J], Transactions of China Electrotechnical Society, 2013,28(10): 12-18

[3] Li Yang, Yang Qingxin, Chen Haiyan, et al. Analysis of factors influencing power and efficiency in wireless power transfer system[J]. Advanced Technology of Electrical Engineering and Energy, 2012, 31(3): 31-34, 39 
[4] S. Li, W. Li, J. Deng, et al. A Double-Sided LCC Compensation Network and Its Tuning Method for Wireless Power Transfer[J], IEEE Transactions on Vehicular Technology,2014,64(6): 62261-62273

[5] C.-S. Wang, O. H. Stielau, G. A. Covic. Design Considerations for a Contactless Electric Vehicle Battery Charger [J], IEEE Transactions on Industrial Electronics, 2005, 52(5): 1308-1314

[6] Sun Yue, Wang Zhihui, Dai Xin, et al. Study of Frequency Stability of Contactless PowerTransmission System [J]. Transactions of China Electrotechnical Society,2005, 20(11): 56-59

[7] M. Budhia, G. A. Covic, J. T. Boys. Design and Optimization of Circular Magnetic Structures for Lumped Inductive Power Transfer Systems [J], IEEE Transactions on Power Electronics,2011,26(11): 3096-3108

[8] T.-D. Nguyen, S. Li, W. Li, et al. Feasibility study on bipolar pads for efficient wireless power chargers[C]. Applied Power Electronics Conference and Exposition (APEC), 2014. 1676 - 1682.

[9] Li Yang, Yang Qingxin, Yan Zhuo, et al. Analysis on Effective Range of Wireless Power Transfer and Its Impact Factors[J]. Transactions of China Electrotechnical Society, 2013, 28(1): 106-112

[10] G. A. J. Elliott, S. Raabe, G. A. Covic, et al. Multiphase pickups for large lateral tolerance contactless power-transfer systems[J], IEEE Transactions on Industrial Electronics,2010,57(5): $1590-1598$

[11] GrantA.Covic, J. T. Boys, M. L. G. Kissin, et al. A Three-Phase Inductive Power Transfer System for Roadway-Powered Vehicles [J], IEEE Transactions on Industrial Electronics,2007,54(6): 3370-3378

[12] T. Hata, T. Ohmae. Position detection method using induced voltage for battery charge on autonomous electric power supply system for vehicles[C]. Advanced Motion Control, 2004. 187-191.

[13] J. L. Villa, J. Sallan, J. F. S. Osorio, et al. High-Misalignment Tolerant Compensation Topology for ICPT Systems [J], IEEE Transactions on Industrial Electronics,2012,59(2): 945-951

[14] Tan Linlin, Huang Xueliang, Zhao Junfeng. Optimization Design for Disc Resonators of a Wireless Power Transmission System [J]. Transactions of China Electrotechnical Society, 2013, 28(8): $1-6$

[15] W. Zhang, JeffC.White, RajeshK.Malhan, et al. Loosely Coupled Transformer Coil Design to Minimize EMF Radiation in Concerned Areas [J], IEEE Tranctions on Vehicular Technology, 2016, 65(6): 4779-4789

[16] Li Junfeng, Liao Chenglin, Wang Lifang, Decoupling Method of Maximum Efficiency and Transferring Power for Electric Vehicle Wireless Charging System via LCCL Circuit[J], Transactions of China Electrotechnical Society,2015,30: 199-203

[17] M. Budhia, J. T. Boys, G. A. Covic, et al. Development of A Single-sided Flux Magnetic Coupler for Electric Vehicle IPT Charging Systems[J], IEEE Transactions on Industrial Electronics,2013,60(1): 318-328 